\title{
EL PANTANAL: UN EJEMPLO DEL IMPACTO GEOMORFOLOGICO SOBRE EL AMBIENTE
}

\author{
THE PANTANAL: AN EXEMPLE OF GEOMORPHOLOGICAL IMPACT \\ ON THE ENVIRONMENT \\ Jean Tricart \\ Centre de Géographie Appliquée Université Louis Pasteur \\ Strasbourg
}

\begin{abstract}
The Pantanal trough is located at the border line of the "couverture tabulaire" at Mato Grosso Plateau, under contral of faults and Precambrian metamorphic and crystalline continental crust with Apalachian Eocambrian sedimentary landforms. Modern alluvial formations have been formed at least $200 \mathrm{~m}$ below present sea level, having around $300 \mathrm{~m}$ thickness. Thus, the Pantanal is a tectonic trough, completely bordered by the $200 \mathrm{~m}$ contour line. Subsidence explains gentle slopes, which are lesser than 1\%. At the sheet flood fan axe. River floods are running slowly from neighbor mountains areas, allowing a grassland support for more than 5.000 .000 of cattle.

Pantanal alluvial deposits have built enormous coalescent sheet flood fans. The largest one is Taquari, which cross over residual mountain chains at the right hand border of the Paraguay, covering "glacis" (bahadas) lying at its ground. On the contact between this sheet flood fans and the "glacis" there is a series of receptacle lakes, which are fed by the Paraguay floods. Some lakes restore water to the Paraguay River during its low level after floods.

During recent Quatemary dry periods the high Paraguay basin was endorreic. So, a number of Pantanal sheet flood fans are digged by wind erosion, forming troughs. These troughs are partly covered by lagoons and partly by amphibious vegetation, thus increasing runoff deficit. Therefore, the Pantanal plays an important role on the Paraguay hidrology, emphasizing floods but increasing runoff deficit.

It has been suggested to build large dams to regulate water flow in the rivers, near their entrances into the Pantanal. However, this radical change in environmental management needs a lot of knowledge, specially on this very complex and feeble environment, where, as a consequence, investments are very ex pensive.
\end{abstract}




\section{RESUME}

La dépression du Pantanal se place au contact de la couverture tabulaire (gres, argilites, quartzites) du Plateau du Mato Grosso, affectée de failles (fig. 1) et du socle précambrien metamorphique et cristallin (fig. 4), avec des reliefs appalachiens sédimentaires éocambriens (fig. 2). Les formations alluviales récentes descendent bien en dessous du niveau marin actuel, jusque vers $200 \mathrm{~m}$ au moins et dépassent par endroits $300 \mathrm{~m}$ d'épaisseur. Le Pantanal est donc une fosse tectonique. II est entièrement englobé dans la courbe de $200 \mathrm{~m}$. L'affaissement explique la faiblesse des pentes, inferieures ò $1 \%$ dans l'axe des épandages alluviaux. Les crues arrivant des reliefs bordiers s'écoulent très lentement. Les inondations provoquent des pertes élevées dans un troupeau de 5.000.000 de bovins.

Les accumulations alluviales du Pantanal forment d'énormes épandages coalescents. Le plus vaste celui du Taquari, pénètre entre les chaînes rési. duelles de la rive droite du Paraguay et recouvre les glacis faconnés à leur pied. Au contact de cet épandage et des glacis, une série des lacs recoivent par des défluents les eaux de crue du Paraguay (fig. 4). Certains de ces lacs restituent de l'eau au Paraguay lorsque son niveau baisse après la crue.

Lors des périodes sèches du Quaternaire récent, le haut bassin était endorreique. De nombreux épandages du Pantanal sont troués de cuvettes de déflation éolienne (figs. 2 et 3 ). Les cuvettes occupées en partie par des étangs, en partie par de la végétation amphibie, accroissent qussi le déficit d'écoulement. Ainsi, le Pantanal joue un rôle considérable dans l'hydrologie du Paraguay: il écrête les crues maîs accroit le déficit d'écoulement.

On a pensé à édifier des barrages sur les cours d'eau près de leur débouché dans le Pantanal. Mais, un tel changement radical de mise en valeur du territoire ne sera pas possible avant longtemps du fait qu'il manquent des connaissances sur ce milieu complexe et fragil et que les investissements seraient très élevées. 


\section{INTRODUCCION}

En el corazón de América del Sur, donde se unen las fronteras de Brasil, Bolivia y Paraguay, el Pantanal es todavía una región poco poblada, de economía pastoral extensiva, que plantea difíciles problemas de ordenamiento. Su solución requiere un conocimiento científico mucho más elaborado que los elementos dispersos e incompletos de los cuales disponemos actualmente (ALMEIDA \& LIMA, 1956; MINISTERIO DAS MINAS E ENERGIA, DN PM，1976; SILVESTRE \& ROMEU, 1974; VALVERDE, 1972). (1)

El Pantanal, como lo indica claramente la etimología de su nombre, es una región anfibia, con muchas lagunas, lagunetas y lagos, con extensas áreas inundables, donde, frecuentemente, los cauces de los ríos son inestables y hasta mal definidos. El Pantanal pertenece a la cuenca del rio Paraguay. Su extensión, en el territorio brasileño alcanza los $110.000 \mathrm{~km} 2$. Su principal riqueza es la cria, en casi libertad, de unos 5.000 .000 de bovinos, actividad altamente perjudicada por las inundaciones. Por otro lado, un serio freno al desarrollo económico es la consecuencia de las dificultades de comunicación. La vía de ferrocarril estrecha (1 $\mathrm{m}$ ) que une Bolivia a Sao Paulo y Santos pasa por Corumbá, en el S del Pantanal, pero no permite un tráfico intenso y de bajo costo. La navegación sobre el río Paraguay hasta la Plata es muy larga y sobre todo, muy difícil. El calaje de los barcos es limitado a $0,8 \mathrm{~m}$. En 1979, cuando una crecida excepcional cortó la línea del ferrocarril, Corumbá se comunicaba esencialmente por vía aérea con el resto del Brasil.

Estas dos dificultades mayores que limitan el desarrollo del Pantanal, las dificultades de navegación sobre el río Paraguay y el regimen hidrológico sin controlar de la región, resulta ambas de la evolución geomorfológica y de las importantes herencias paleoclimatológicas involucradas en ella (KLAMMER, 1981).

\section{EL PANTANAL EN EL MARCO REGIONAL}

A pesar de su alejamiento del mar, el Pantanal es un área muy deprimida en altura. Al dejarlo, el río Paraguay se encuentra a menos de $80 \mathrm{~m}$. Casi todo el Pantanal está bajo los $200 \mathrm{~m}$ y una buena extensión de él a menos de $100 \mathrm{~m}$ de altura. Es una extensa llanura, muy plana, que es la raíz de los problemas hidrológicos. Cuiabá, aguas arriba del Pantanal, se encuentra a sólo $150 \mathrm{~m}$ sobre el nivel del mar. La inclinación general de la depresión es del $E$ hacia el $W$ con una débil pendiente del $\mathrm{N}$ al $\mathrm{S}$, a lo largo del río Paraguay. Esta disposición hace que este rio margine prácticamente el Pantanal al W. A lo largo de su cauce se observan en la frontera Brasil - Balivia y en Bolivia, extensas explana das que dominan sierras aisladas del tipo inselberg (sierras-islas). Al $\mathrm{N}$ y al $\mathrm{S}$, se mantiene la misma asociación de formas, pero con sierras más extensas y más altas, de disposición más diversa. Ellas cierran la depresión del Pantanal y estrangulan el valle del rio Paraguay cerca de su salida del Brasil. Al E, finalmente, la llanura anfibia es marginada bruscamente por una escarpa de 300 a 400 m de alto, rectilinea, sin disectar, impropiamente llamada Sierra de Maracaju (fig. 1). Se trata, en realidad, de la extremidad de los planaltos del Mato Grosso

(1) La alta cuenca del ría Paroguay ha sido objeto de un programa de investigación, apo. yado inicialmente por la UNESCO y, muy rápidamente objeto del interés del PNUD y de la OEA. Pero, este programa fue orientado hacia la elaboracion de un modelo matemático de hidrología, lo que ero muy prematuro, tomando en consideración la escasez de conocimientos básicos. La labor desempeñada en esta oportunidad dió lugar a una publicación del Ministerio del Interior (MINTER, 1966-1972) que hemos cabalmente utilizado. 


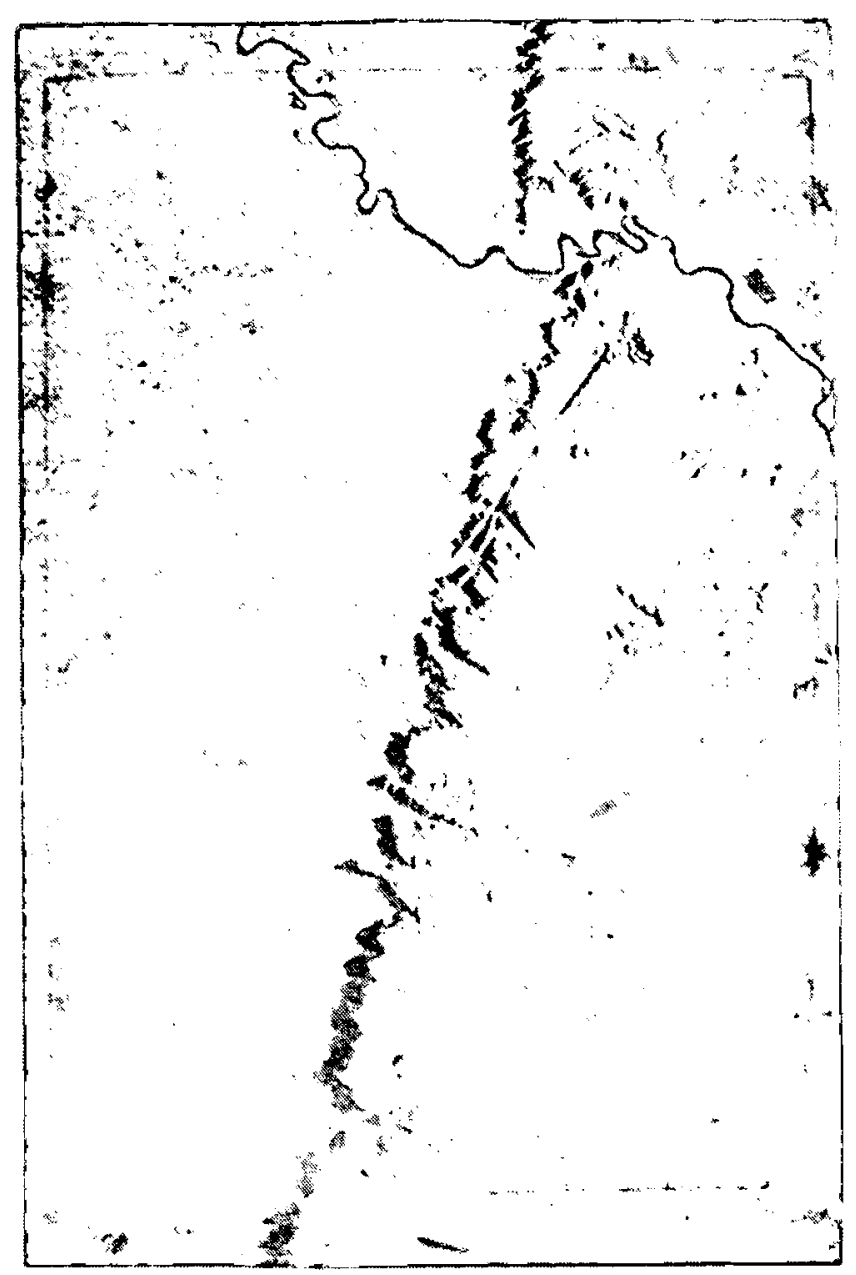

1. El escarpe oriental del Pantanal. Mosaico de radar. Desembocadura del río Taquari en la depresión cerca de Coxim. Apice del abanico del rio Taquari. Es. carpe de falla monoclinal, con disposición de relieve de cuesta. Numerosas fajas de trituración probablemente asociadas con fallas en la parte marginal del Planalto Matogrossense.

del $S$, que se siguen por centenas y centenas de kilómetros hasta el W del Estado de Soo Paulo, cortados por valles encañonados y subdivididos por una serie de escalones menores. Ellos, como el escalón marginal, se orientan del $\mathrm{N}$ al $\mathrm{S}$. El Pantanal es, en consecuencia, una depresión bien definida del lado $E$, pero que paso paulatinamente a otras unidades topográficas en sus otros mórgenes.

Esta disposición orográfica es una consecuencia de la estructura geológica. El Pantanal, es en realidad, hasta cierto punto, una depresión marginal de contacto entre una plataforma precámbrica y su cobertura sedimentatia discordante. Esta forma del planalto oriental consiste en formaciones del fin del Paleozoico y del Mesozoico, integradas por alternancias tabulares de areniscas, cuarcitas y lavas volcánicas intrusivas (sillas y trapps). Ellas buzan suavemente en dirección este. Los escalones del Planalto Mato Grossense son curestas, asi como un 
tramo, al $N$, del escalón marginal del Pantanal. Pero, al $S$, donde su trazadóes más recto, este escalón es un abrupto de falla. Al $S$ y al W del Pantanal, aflora el basamento precámbrico, intensamente plegado y metamorfizado $y$, por consiguiente, de litología muy heterogénea. Cuarcitas y metavulcanitas ofrecen una resistencia al intemperismo mayor que los granitos y gneisses. Asi mismo, ellos conservan en forma de relieves residuales, sierras-islas y cerros-islas. Al $\mathrm{N}$ y NE, sobre el basamento, una serie sedimentaria del Precámbrico superior y del Paleozoico inferior - integrada por conglomerados, dolomías, areniscas, arcillas y siltitas con estratificaciones peliculares ("folhelhos" de los geólogos brasileños) y calizas - ha sufrido plegamientos de cobertura, amplios y regulares, de tipo apalachiano. Predominan los sinclinales colgados en sistemas de crestas monoclinales y barras, con algunas manifestaciones cársticas (MENDES, 1957). Al pie de estos relieves se extienden explanadas en parte recubiertas por los aluviones de los ríos (Cuiabá, Poconé, Bento, Gómez). Los más típicos relieves apalachianos los forman las Serra de Chacororé y Serra dos Araras (fig. 2) Al ENE de Cáceres, la fuente cárstica llamada Fonte Progresso, tiene un caudal de $9.00 \mathrm{~m} 3 / \mathrm{h}$. Una descarga tan abundante revela una carstificación muy intensa, sobre todo por afectar rocas bastantes antiguas $y$, por eso, poco porosas y muy recristalizadas, de corrosión lenta. Esta carstificación es necesariamente antigua y atestigua condiciones relativamente húmedas.

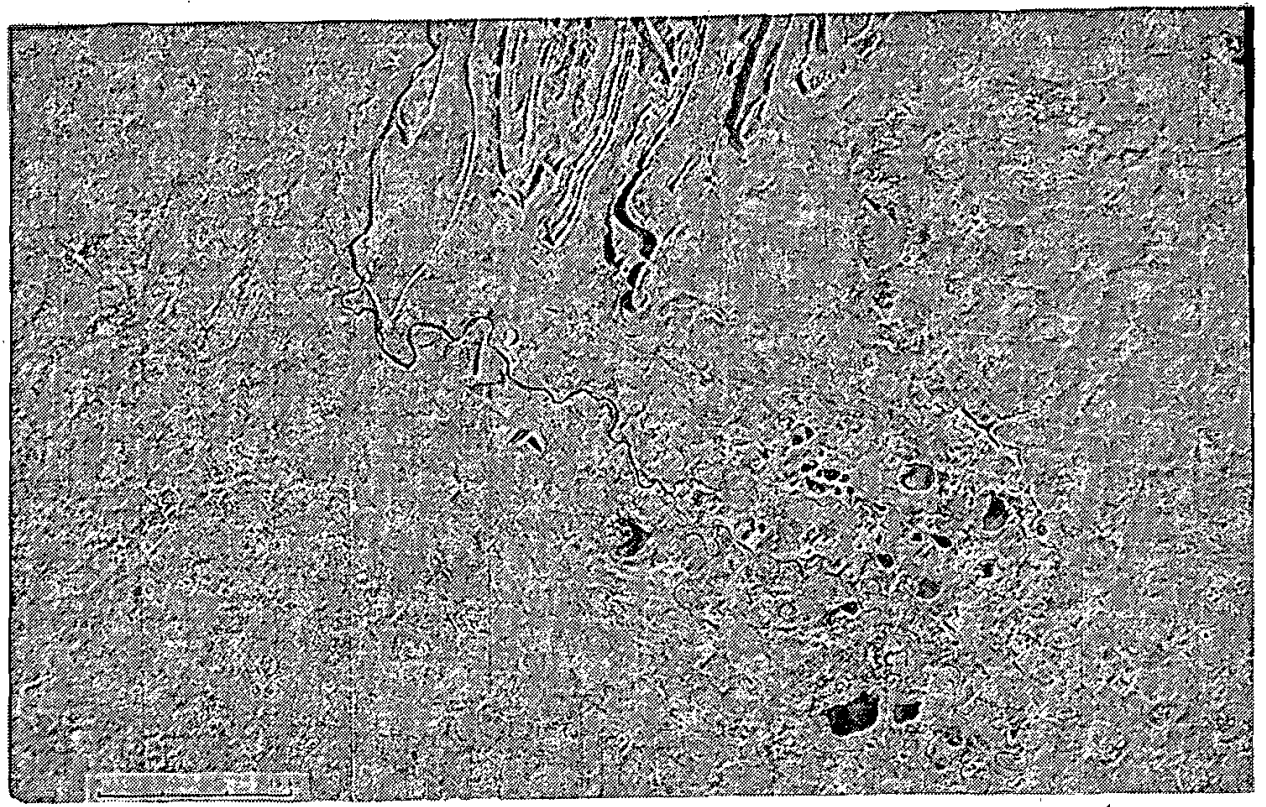

2. Margen Norte del Pantanal a lo largo del cauce del alto Paraguay. Mosaico de radar. Relieves apalachianos de las capas plegadas del Eocámbrico y del Paleozoico de la Serra dos Araras, lecbo meándrico y desparramadero del rio Paraguay, con extensas cubetas lacustres, excavadas por el viento durante el último periodo seco. Al NE, desparramadero del río Cuiabá, que se une con aquél del río Paraguay.

Pero, las condiciones estructurales que acabamos de describir no explicán perfectamente la existencia $y$, sobre todo, la extensión del Pantanal. En efecto, tanto al $N$ como al $S$ y al $W$, las sierras están rodeadas de explanadas que se hunden suavemente por debajo de las inmensas acumulaciones aluviales donde se observa las ciénagas. 
Los relieves residuales desaparecen rápidamente después de haber dismi nuido de altura al acercarse al margen del Pantanal. Al E, ya hemos señalado un escarpe de falla. No puede ser muy antiguo por su mínima disección, incluso si se toma en cuenta la resistencia alta de las rocas (cuarcitas y lavas voleánicas). Valles cortos, de pocos kilómetros de largo, muy estrechos, con fuerte inclinación lo cincelan. Los ríos importantes y mós caudalosos del Pantanal, no han cortado más que valles encañonados, a pesar de presentar un trazado anguloso que hace sospechar su adaptación a alineamientos tectónicos y fajas de trituración lo que explica que el río Coxim, por ejemplo, desemboque muy ablicuamente, de "soslayo", podría decirse, en el Pantanal (fig. 1).

En su margen oriental, al $S$ del río Taquari, el Pantanal ofrece claramente el aspecto de un bloque hundido al pie de un escalón de falla reciente, muy probablemente cuaternario.

La información geológica relativa al basamento del Pantanal es escasa. Se hicieron, pues, algunas perforaciones de exploración petrolera. Sin embargo, el registro carece de precisión y pormenores sobre el material que lo cubre. A pesar de eso, puede comprobarse el gran espesor de estos terrenos. En el margen del inmenso abanico aluvial del rio Taquari, a $75 \mathrm{~km}$ ESE de Corumbá, la Formación Corumbá que aflora en la ciudad y forma los cerros que la dominan, ha sido encontrada a $130 \mathrm{~m}$ de profundidad. Es muy probable que sólo el Cuaternario la recubra. En este caso, la base del Cuaternario se encontraría $30.40 \mathrm{~m}$ debajo del nivel del mar. Más al $N$, no rivy lejos del centro geométrico del mismo abanico, en la Fazenda San Sebastião, el basamento culmina a 227 m debajo de la superficie, es decir por lo menos a $-120 \mathrm{~m}$. En la Fazenda Piquiri, al $\mathrm{N}$ del río del mismo nombre, no se ha encontrado todavía el basamento a $420 \mathrm{~m}$ de profundidad, es decir a $-300 \mathrm{~m}$. En el abanico del río Taquari, pero más al $\mathrm{S}$, al WSW de Uniao (Faz San Bento), la perforación alcanzó a $320 \mathrm{~m}$ sin haber encontrado la base del Cuaternorio. Esta tiene que ubicarse a una cota inferior a $-180 \mathrm{~m}$.

Estos datos no permiten pormenorizar la tectónica del Pantanal, pero autorizan a afirmar que este es una fosa de hundimiento. Su margen oriental consiste en una serie de escalones de fallas "en échelon". A.I N, al W y al S, se trata de un basculamiento suave, lo que provoca el recubrimiento de las explanadas, probablemente anteriores al Cuaternario, por los grandes derrames aluviales en forma de abanicos. En el $N$, donde afloran calizas, estas explanadas han sido perforadas por numerosas dolinas, ahogadas en el presente. Cuando fueron excavadas, las explanadas tenían que encontrarse algo más altas que el acuífero: es decir que el centro del Pantanal estaba necesariamente a una altura menor que ellas. El ahogamiento posterior de las dolinas sería la consecuencia de la acumulación de una cantidad enorme de material aluvial desencadenada por el hundimiento. El Pantanal sería un área afectada por subsidencia durante el Cuaternario. Es posible que el relleno cuaternario esconda algunas fallas pero más bien nos parece que el Pantanal es una depresión de ángulo de falla, en forma de sinclinal muy abierta de eje W-E, buzando para $E$ y cerrada al $E$ por una serie de fallas aproximadamente N-S. La subida tectónica hacia el $S$ debe haber cerrado la depresión cuyo fondo está bajo el nivel del mar. El rio Paraguay ha sido obligado a cortar el basamento vasculado y levantado al $S$ de la fosa, lo que explica la poca profundidad de su lecho y las dificultades que ello ocasiona para la novegación.

El Pantanal parece integrarse en la fajo de contacto entre el escudo brasileño y el sistema andino. La orogénesis andina, caracterizada en el $N$ de Chile y el $S$ del Perú por un paroxismo pliocuaternario, tuvo relación con una fragmento- 
ción del margen del escudo en una serie de bloques desnivelados. El Pantanal es uno de ellos.

La tectónica explica la baja alfitud de una larga faja de territorio al E de los Andes, desde el río de la Plata hasta el S de Amazonia, pasando por el Chaco argentino y el Pantanal. Esta disposición orográfica influye sobre el clima, incidiendo particularmente en la migración, varias veces por siglo, de masas de aire frío patagónico hasta el $S$ de Amazonia. Los brasileños las llaman "friagens". Acompañadas de lluvias y de viento, ellas pueden hacer bajar la temperatura incluso hasta los 10, en río Branco, capital del Estado de Acre, a sólo $10^{\circ}$ de latitud S. Después de pasar el frente frío, el tiempo se torna muy claro. Por otra parte, masas de aire tropical húmedo pueden llegar desde el NE al Pantanal, caracterizadas por fuertes lluvias, principalmente sobre el Planalto Mato-Grossense, drenado por el río Taquari y por parte del río São Lorenzo que lo inundan.

El clima actual del Pantanal se caracteriza por una cierta inestabilidad, como consecuencia de los dos principales fenómenos que acabamos de recordar. A pesar de ello, puede clasificarse como clima tropical, con una estación seca durante el invierno austral, lo que explica la vegetación de bosque espinoso y denso, xeromórfico, del Chaco, que se encuentra al $W$ y al SSW del Pantanal. Al $E$, el Planalto Mato-Grossense entra en el área de los "cerrados" del Brasil central, es decir, una formación vegetal caracterizada por un estrato muy abierto de árboles de unos $10 \mathrm{~m}$ de alto, con un estrato arbustivo denso en el que se encuentran algunas especies xeromórficas $y$, finalmente, un estrato bajo de matorral bajo y plantas herbáceas. Al N, en pocas centenas de kilómetros, se pasa del cerrado a la selva tropófila y a la selva amazónica. En el propio Pantanal, las lluvias alcanzan una media anual del orden de $1.500 \mathrm{~mm}$ en el margen NE, de 1.250 en el margen $S$ y un mínimo de $800-900 \mathrm{~mm}$ en los alrededores de Castelo y de la confluencia del río Cuiabá y del río Paraguay. Estas no son, de ninguna manera, condiciones subáridas. Pero, por la característica del ambiente, el regimen hídrico está más influído por la hidrología de los ríos que por las precipitaciones.

Para entender la hidrología del Pantanal, debemos examinar sus acumulaciones aluviales.

\section{LAS ACUMULACIONES ALUVIALES}

El patrón de la red hidrográfica del Pantanal parece paradojal. Acabamos de mostrar que la fosa de hundimiento del Pantanal es basculada hacia el E. Ahora bien, el río Paraguay bordea su margen occidental, muy cerca del contacto entre las acumulaciones aluviales y las explanadas cortadas en el basamento. La depresión del Pantanal es recorrida por afluentes nacidos en los relieves más altos que la rodean. La disimetría de la red es notable: el rio Paraguay recibe todos los afluentes importantes en su margen izquierdo: río Cuiabá, con sus afluentes, los ríos San Lorenzo, Piquirí y Cuiabá Mirim; rio Taquari, río Aquidauana que se une con los ríos Nioaque y Miranda. En el margen derecho, los únicos afluentes perennes son los de aguas arriba, el rio Cabacal y la corixa Grande. Este último, en realidad, es más una ciénaga que un río en su cauce inferior, lo que significa "corixa": vegetación de pantano con un movimiento muy lento de un espejo de agua de nivel bastante fluctuante. Todos los demás afluentes del margen derecho, o son "canales", es decir, brazos con corrientes alternativas que unen un río y un lago o dos lagos entre sí, o son arroyos con escorrentia espasmódica, funcionando como "guadis". Esto tiene su explicación en el clima más seco del Chaco boliviano y paraguayo. 
La llanura del Pantanal se presento muy débilmente inclinada de $\mathrm{E}$ a W. En el centro de la fosa, por ejemplo, el margen oriental se encuentra aproximadamente a $190 \mathrm{~m}$ de altitud cerca de Coxim y del río Taquari, y las orillas del río Paraguay en Porto San Francisco, a $91 \mathrm{~m}$. El desnivel es de $100 \mathrm{~m}$ sobre una distancia en línea recta de $280 \mathrm{~km}$., es decir, una pendiente media de $0,035 \%$ o unos $35 \mathrm{~cm}$ por $1 \mathrm{~km}$. A causa de ello podemos entender la falta de encauzamiento y la gran extensión de las inundaciones. En el $N$, el panorama es semejante a lo largo del río Cuiabá, la vega está a $120 \mathrm{~m}$ de altitud en Retiro Biguacal a unos $30 \mathrm{~km}$ del pie de la Serra da Chacororé. La confluencia en el río Paraguay se encuentra a $91 \mathrm{~m}$. La distancia, siempre en línea recta es de 220 $\mathrm{km}$, lo que indica una inclinación media de sólo $0,0136 \%, 013,6 \mathrm{~cm}$ por $\mathrm{km}$. Lo mismo se observa en el $\mathrm{S}$, a lo largo de las vegas de los ríos Aquidauana y Miranda, cuya altitud es de $147 \mathrm{~m}$ en Aquidavana y de $85 \mathrm{~m}$ en la confluencia con el río Paraguay, a unos $220 \mathrm{~km}$ de Aquidauana (pendiente media $0,028 \% \circ 28$ $\mathrm{cm} / \mathrm{km}$ ). La pendiente es algo superior en el centro de la depresión que en sus extremos $\mathrm{N}$ y $\mathrm{S}$.

Estas características topográficas son la consecuencia de la disposición de las acumulaciones aluviales. El Pantanal consiste en una serie de inmensos abanicos aluviales coalescentes, de pendiente sumamente débil. El perfil CoximPorto Soo Francisco coincide aproximadamente con la generatriz central del abanico del río Taquari, debido a que allí la inclinación es relativamente fuerte. Se trata de una "cumbre" de abanico, más alta que las faldas laterales. El propio río Taquari no corre a lo largo de este eje de abanico, sino con un trazado curvo, a lo largo de su margen derecho. Este tipo de disposición se encuentra frecuentemente sobre los conos de deyección, pero con una topografía más acentuada y sobre áreas de superficie mucho más reducida. Es por esto que no nos parece conveniente llamar estas acumulaciones "conos de deyección" lo que daría una impresión falsa de su topografía. Por eso las llamamos abanicos aluviales.

Los abanicos aluviales tienen sus ápices en el piedemonte de las sierras del Precámbrico, generalmente en débiles concavidades de la topografía, generadas por la coalescencia de dos explanadas de inclinación distinta, apoyadas sobre relieves residuales diferentes. Hacia abajo, se pasa de una simple vega que ocupa el fondo de un valle definido, a un abanico sin valle, que se ensancha paulatina pero rápidamente. Por ejemplo, el abanico construido en conjunto por el alto río Paraguay y el río Jauru empieza en Cáceres, donde confluye el río Cabacal, tiene una superficie, mayor que $7.500 \mathrm{~km} 2$. Su ancho, en linea recta llega a unos $140 \mathrm{~km}$ en su extremidad baja. Al S, el abanico del río Miranda aguas abajo del puente del ferrccarril, tiene uno longitud de $150 \mathrm{~km}$; el del río Aqui dauano, también aguas abajo del ferrocarril, cerca de $130 \mathrm{~km}$. En conjunto, cubren entre 9.300 y $9.500 \mathrm{~km} 2$, teniendo en cuenta la incertidumbre respecto a sus límites. Los desparramaderos edificados conjuntamente por los ríos Cuiabá y Sao Lorenzo tienen una superficie de $27.000-28.000 \mathrm{~km} 2$, mucho mayor que la del abanico del alto rio Paraguay. Pero, la mayor parte del Pantanal, la ocupa el desparramadero (sheet flood area) del río Taquari, cuya generatriz mide 150 $\mathrm{km}$ de longitud y cuya base a lo largo del río Paraguay, unos $275 \ldots$

Estos desparramaderos (sheet flood area) en forma de abanicos no son activos en el presente. Los cubre una vegetación densa de bosques cienagosos y de sabanas arboladas. Muchos cauces se observan en su superficie, pero ellos son discontinuos. Salen de una depresión más húmeda, que se inunda durante las crecidas. Forman una cuneta muy suave y poco honda, pero más húmeda que los alrededores $y$, por eso, colonizada por una vegetación distinta, que puede iden- 
tificarse muy bien sobre los mosaicos de radar por su reflectividad fuerte. En período de aguas altas o de inundación, una cierta escorrentía, muy lenta, se produce en ella. El nombre que se le da en el Pantanal es "vazante" o "corixa". (fig. 3). Corixas y vazantes son también, frecuentemente alimentadas por manantiales localizados a lo largo de rupturas de pendiente de la superficie del desparramadero o más frecuentemente, en sitios donde cambia la facies del material aluvial. En efecto, los abanicos consisten en arena, limos y arcillas con algunas pocas gravillas en la parte alta. La arena fué acumulada en forma de bancos en los cauces o a lo largo de sus orillas. Los materiales finos se depositaron por decantación en cubetas. Frecuentemente un brazo termina en forma de pequeño delta en una cubeta. El agua infiltrada en su material arenoso se encuentra representada en su extremidad, par la desaparición de las facies arenosas $y$, por consecuencia, sube hasta la superficie donde ella forma un manantial. Las inundaciones en el ápice de los desparramaderos permiten la alimentación de estos pequeños acuíferos. Pero, pocas, muy pocas, son las "vazantes" que tienen una continuidad suficiente para confluir en un río. La mayoría de ellas se pierde paulatinamente aguas abajo. La cuneta suave en la cual han nacido desaparece. El escurrimiento del agua, sobre pendientes tan débiles, es tan lento que el agua se pierde por evaporación y por la transpiración de la abundante vegetación que ocupa la "vazante". El déficit de escurrimiento debe ser enorme...

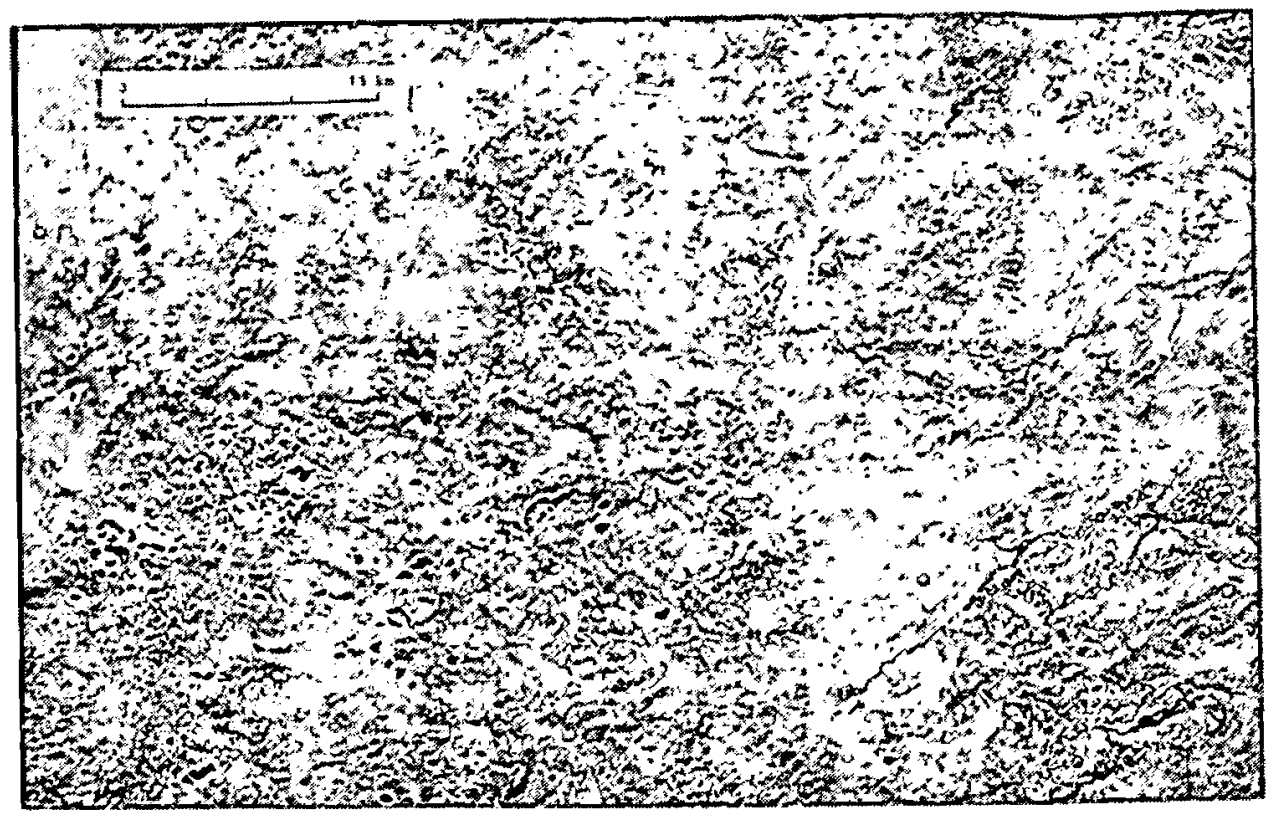

3. Parte centro-sur del abanico del río Taquari. Mosaico de radar. Al E, una serie de cauces, con agua, visibles en negro sobre la imagen, son alimentados por manantiales. Ellos se pierden bacia el W. Sus lecbos son mal calibrados: se trata de formas relictas. En el NW, serie de corixas, en las cuales no bay agua libre. Ellos se notan por la alta reflectividad de la vegetación anfibia que en ellos crece. En el centro y $\mathrm{SW}$, numerosas cubetas de deflación, algunas con agua libre, otras con vegetación anfibia.

Las crecidas provocan desbordamiento de los ríos debido a que sus orillas son bajas y discontinuas y que, por otra parte, luego que ellos llegan al área subsidente, su perfil longitudinal se caracteriza por una inclinación sumamente 
débil. Las crecidas son frecuentemente bruscas cuando llegan al Pantanal, principalmente las de los ríos encajonados del Planalto Mato-Grossense, como consecuencia del encañonamiento, pero rápidamente por la falta de pendiente, se desparraman. Las selvas de galería, muy desarrolladas en lo parte de arriba de las vegas, disminuyen la violencia de los desbordamientos, que pasan a tomar la forma de láminas de inundación. También, estos bosques de galerias, por la frenada de la corriente (efecto de rugosidad)) provocan una importante decantación del material en suspensión. Por esto, los desparramadores actuales no edifican abanicos aluviales. Sólo una pequeña cantidad de material fino se deposita en las depresiones, donde se mezcla con muchos detritos vegetales, generando un material turboso. Los desparramaderos ya no son formas activas, sino que, posiblemente, formas relictos. A pesar de ello, siguen desempeñando un papel hidrológico de suma importancia.

Debemos investigar, pues, las condiciones en las cuales se edificaron estos enormes abanicos aluviales, condiciones que fueron, necesariamente, diferentes a las actuales.

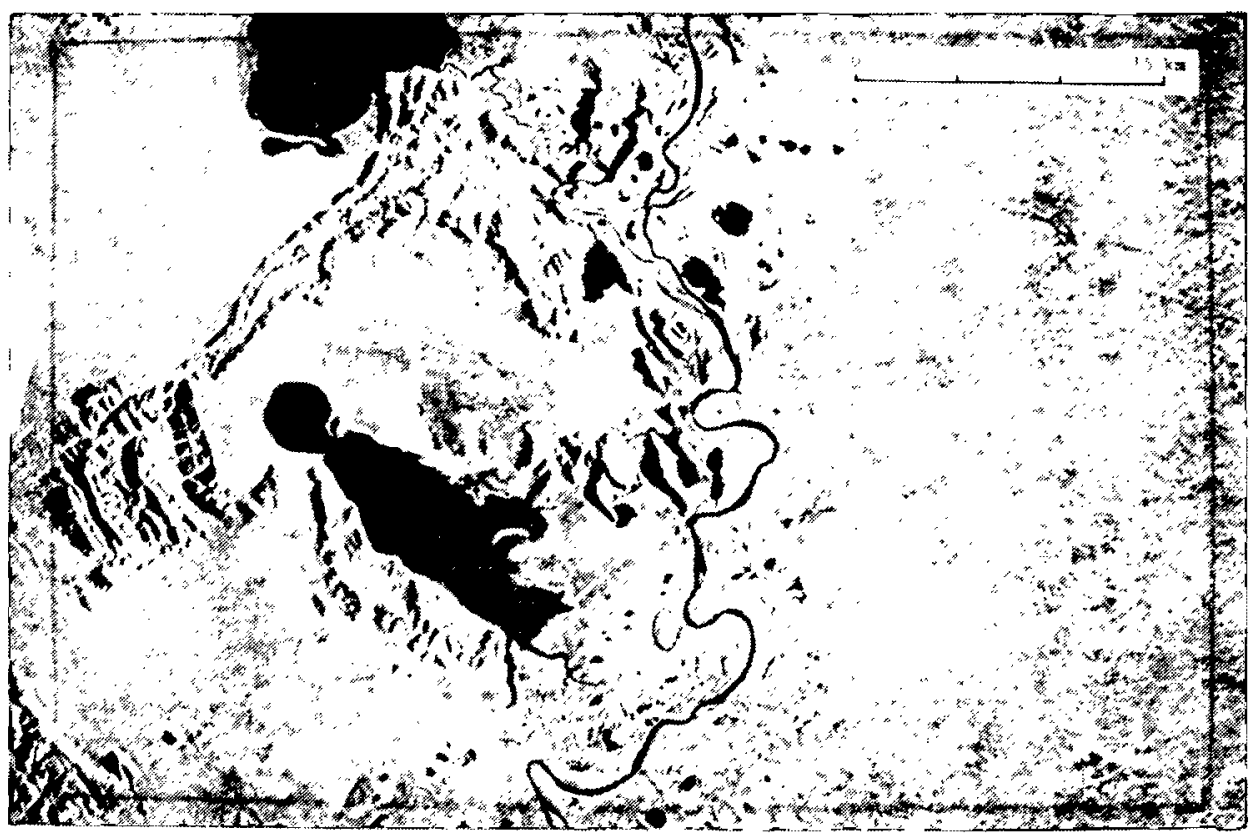

4. Margen occidental del Pantanal, mosaico de radar. Al l:, la parte baja del río Taquari, sus depósitos penetran entre las sierras del Precámbrico metamórfico y cristalino. Al $W$, en Bolivia, en el metamórfico, la alteración diferencial y la disección posterior evidencian la disposición plegada del material. Esta serranias-islas dominan explanadas que recubre el bosque seco chaqueño. Los derrames del Taquari ban penetrado entre las serranias y recubren una parte de estas explanadas, lo que crea contra-pendientes. Asi se explica los lagos (Laguna do Castelo al S, Laguna Mandioré al N).. Se observa muy claramente: un gran desparramadero con vegetación anfibia al $N$ de la Laguna do Castelo; otro al SE; el canal que une el rio Paraguay con la Laguna Mandioré; otro que desemboca en el extremo SE de la Laguna do Castelo; los meandros del río Paraguay y los albardones evidenciados por la vegetación. 


\section{CONDICIONES PALEOGEOMORFOLOGICAS EN EL PANTANAL}

La extensión enorme de los desparramaderos y su volúmen aún mayor implican:

1. Que grandes cantidades de material detrítico fueron suministradas por las cuencas superiores de los ríos. El Planalto Mato-Grossense presenta un modelado de disección muy intensa con una fuerte densidad de valles en algunas de sus partes, al pie de las cuestas principalmente, donde afloran rocas blandas: areniscas casi sin consolidar, arcillas pelíticas, etc. Es una de las fuentes importantes de acumulación aluvial en el Pantanal. Estas facies ocupan extensiones relativamente grandes en la cuenca del río Taquari, lo que coincide con la magnitud excepcional del abanico de dicho río. Otros se encuentran en las demás cuencas, también edificando derrames de importancia al pie de los planaltos y escalones de la cobertura paleo-mesozoica.

2. Que, por otra parte, la acumulación no era frenada o encauzada en el Pantanal por una cubierta vegetal densa, como es el caso actualmente. El estudio de los mosaicos de radar mostró una cantidad increíble de canales formando una red trenzada sobre casi toda la superficie de los abanicos. Sólo en algunas partes distales de ellos, falta este patrón: se trata en este caso, de decantaciones de cubetas de material fino. Ellas se ubican principalmente en el $S$ y $S W$ del abanico del Taquari y la parte más baja del abanico del alto Paraguay y del río Cuiabá (figs. 2 y 3 ). En estas áreas hay muchos hundimientos excavados por deflación, lo que también obliga a rechazar una cubierta vegetal densa y cerrada.

Estos dos requerimientos permiten concluir que, por lo menos cuando se acumularon los materiales de la parte superior de la acumulación, las condiciones climáticas no permitían el crecimiento de una cubierta vegetal bastante densa para asegurar una fitoestabilización del terreno, sea en las áreas de disección, sea en las acumulaciones. Ahora bien, el Planaltc Mato-Grossense es en el presente, la parte más húmeda de toda la región. Podemos deducir que la aridez debia ser mayor en otras portes. Esta opinión se apoya sobre varias observaciones.

En ciertas partes del Pantanal, puede observarse una gran cantidad de depresiones cerradas, sin desague, de forma semi-circular, con margenes suaves, poco profundas. La mayor parte de las que pueden identificarse sobre los mosaicos de radar, tienen un diámetro del orden de $1 \mathrm{~km}$. Ellas son numerosísimas en la parte distal del abanico del río Taquari en el $\mathrm{S}$ y $\mathrm{SW}$. Otras, generalmente mayores, con diámetros de más de $5.6 \mathrm{~km}$ se ubican en las vegas del cauce inferior del río Cuiabá y del alto Paraguay, y en la llanura entre ellos (fig. 5).

Las cubetas son inundadas algunas de manera permanente, otras sólo durante los períodos de lluvias y crecidas. En época seca, como la de los vuelos de levantamiento de cobertura de radar (1976), muchas cubetas no son inundadas, pero si muy húmedas, con una vegetación cenagosa. Otras, inundadas por agua poco profunda, son totalmente recubiertas de vegetación anfibia, con intensa reflectividad de la onda de radar. Actualmente, todas estas depresiones están obliteradas. Una colmatación de los detritos vegetales, a la cual se añade, en ciertos casos, material fino de decantación traído por la llegada de aguas de inundación, $y$, en algunos pocos casos, divagaciones de brazos de ríos, son los procesos que, paulatinamente, generan esta obliteración.

En el $S$ del abanico del río Taquari, una parte de estas cubetas consiste en suelo desnudo, arcilloso, con concentraciones de sales que cristalizaron por evaporación en las partes más hondas. Se llaman "barreiros" en la comarca. La 


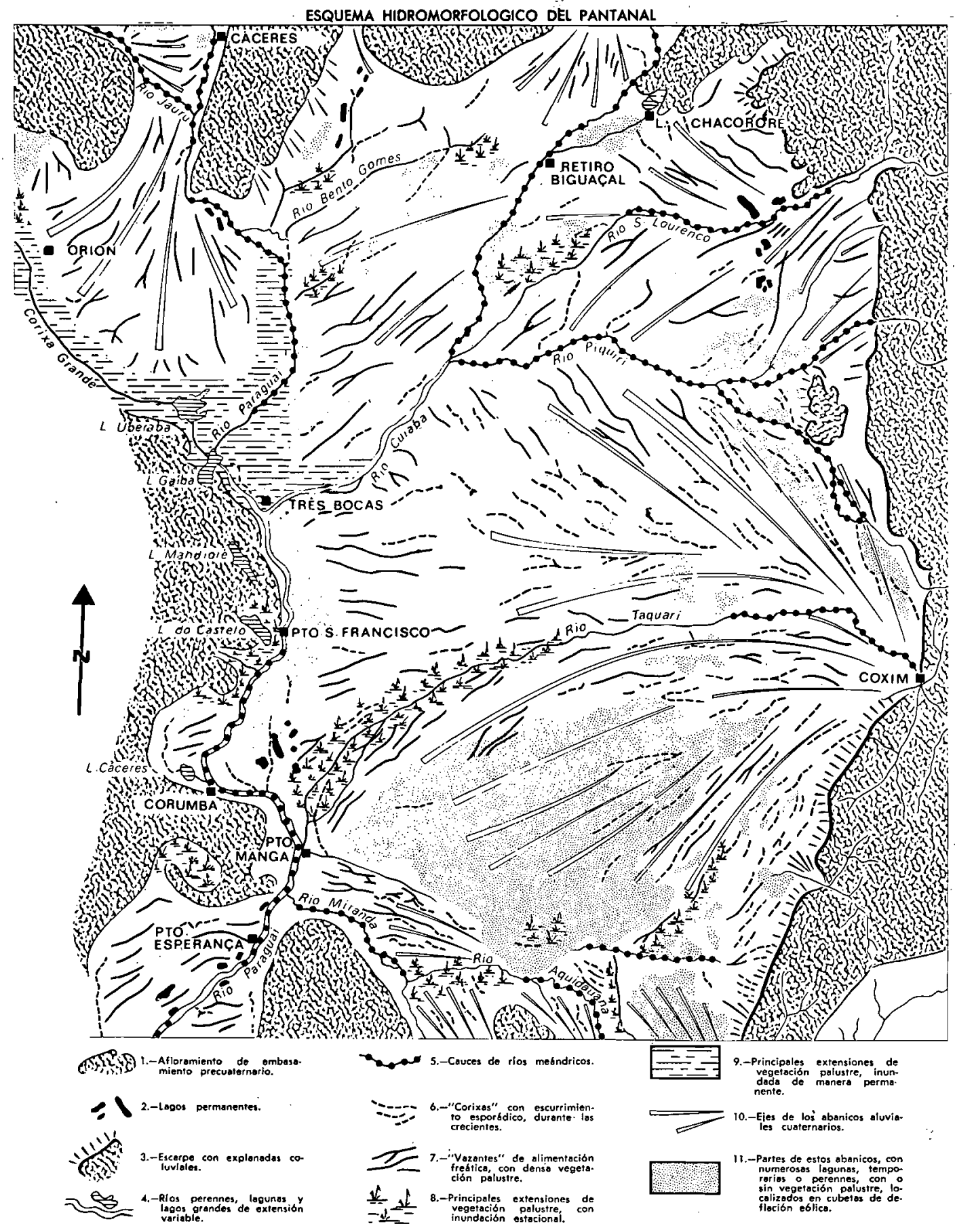

Pig. 5. 
fauna silvestre y el ganado los conocen bien y los frecuentan para lamer el suelo y lograr la cantidad de sal que su organismo necesita. Un geólogo realizando investigaciones de minería (DA CUNHA, 1943) describe los "barreiros", señalando también en sus cercanías, pozos con agua salobre. Cerca de Nhecolandia, en esta área, él midió en una laguneta, un PH de 9 y, en otra, más al $E$, uno de 10, en una laguneta de 5 ha de extensión. Según él, las sales son cloruro de sodio y salitre. El mismo autor indica la presencia de tales lagunas y depresiones saladas en los alrededores de Porto Espiridiao, antiguamente llamado Porto Salitre, en la alta cuenca del río Jauru, en los alrededores de Cáceres, de los ríos Sao Lorenzo y Cuiabá, a lo largo del río Paraguay entre Corumbá y Porto Murtinho. Estas áreas son efectivamente donde hemos identificado depresiones cerradas sobre los mosaicos de radar.

Estas depresiones saladas todavia permanecen desnudas, a pesar de las condiciones climáticas húmedas actuales, por el exceso de sal que perjudica las plantas; las interpretamos como favorables a la deflación élica. Como en la Pampa Deprimida (Rep. Argentina), durante un período reciente más seco, la deflación eólica ha excavado depresiones cerradas en ciertos ligeros hundimientos de la superficie del suelo donde se concentraban las sales y donde, en consecuencia, la cristalización de la sal provocaba una agregación de la arcilla y, sobre todo, de los limos finos. Los agregados cementados por cristales de sal tienen la granulometría de arenas finas, lo que torna fácil la deflación. Estas depresiones son sebkhas, que prueban un período de clima árido. Una breve nota de SANCHEZ (1977) señala la existencia, en la parte distal del desparramadero del río Taquari, al E de Corumbá, la existencia de pequeños cordones, de 2 a $5 \mathrm{~m}$ de alto, alrededor de estas cubetas: es un rasgo típico de los sebkhas.

Al $\mathrm{S}$ del Pantanal, en la región de serranías constituidas por el basamento precámbrico alzado, donde el clima actual es más seco, hemos identificado, sobre imágenes de LANDSAT, una serie de fenómenos de endorreismo:

1. En el Chaco paraguayo, antiguos valles, anchos y poco encajonados, se pierden en pequeños desparramaderos con cubetas terminales de las cuales no sale ningún cauce. Se excavaron probablemente durante un periodo más húmedo, como parte de una red hidrográfica integrada y exorréica. Pero, actualmente, aguas arriba de los desparramaderos, sólo funciona una escorrentía espasmódica. En las cubetas terminales, estas crecidas-relámpago (flash-floods) alimentan lagunas intermitentes. La composición de infrarrojo de la imagen LANDSAT del 22 de julio de 1976, fecha poco diferente del registro de radar, muestra en estas cubetas aguas muy cargadas de sal y de materia en suspensión. El clima actual no es suficientemente húmedo para permitir la organización de una hidrología exorréica. Permanece todavía, de manera relicta, una red endorréica elaborada durante un período árido reciente, probablemente el mismo que permitió la formación de las sebkhas del Pantanal.

2. En el margen brasileño del río Paraguay, se encuentra una mesa baja cubierta por el mismo tipo de vegetación que en el Chaco paraguayo. Pueden observarse, al pie de la Sierra de la Bodoquena, en la Reserva India de la FUNAl cerca de Porto Braga, una serie de cubetas cerradas donde se terminan los arroyos bajando de la sierra. En la composición de color LANDSAT, en su orilla puede apreciarse un anillo delgado y discontinuo de vegetación con poca materia verde, probablemente una formación abierta de halófitas.

Las mismas manifestaciones de hidrología semi-árida se observan también en la imagen de LANDSAT contigua al $S$, cerca de los ríos Apa y Tereré y del 
rio Perdido, cuyo nombre es significativo. Los ríos Amaguijá y Córrego se pierden sobre un desparramadero donde las pequeñas cubetas saladas son numerosas.

Más al N, el río Paraguay no recibe ningún afluente notable del Chaco tanto paraguayo como boliviano. Sólo se observan cauces con escurrimiento esporádico que no llegan hasta la llanura de inundación del río Paraguay.

Todo el material aluvial acumulado en el Pantanal viene de sus márgenes $\mathrm{E}, \mathrm{N}$ y $\mathrm{S}$. No ha habido suministro por el margen chaqueño. Durante los períodos secos del Cuaternario, aquél ha permanecido bastante más seco. Lo demuestra claramente la disposición de las acumulaciones aluviales.

Ya hemos señalado que el abanico del alto río Paraguay es relativamente más modesto. En su parte baja, él fue "empujado" en dirección al W por los aportes más abundantes del río Cuiabá, que a su vez, lo fue por el abanico del río Taquari. Todo eso refleja una verdadera jerarquía en la abundancia del aluvionamiento. El propio río Paraguay ha edificado su desparramadero al SW de la Sierra dos Araras, alrededor de Ulberaba, hasta Orion (fig. 2). La Corixa Grande, cuya cuenca es de poca extensión y cuyos aportes detríticos fueron débiles, dibuja una amplia curva marginando el extremo del abanico del río Paraguay. Su perfil longitudinal tiene muy poca pendiente. Por falta de material aluvial, la Corixa Grande no ha podido rellenar su valle en forma de vega: lo ocupan ciénagas,y en su extremo, una laguna, Laguna Ulberaba, representada por el desparra madero del río Cuiabá sobre el margen del cual se estableció el cauce del rio Paraguay.

Aguas abajo, el río Paraguay ofrece un aspecto de corixa. Su cauce se ubica al "pie" (si se puede hablar así, con pendientes tan infimas) de los abanicos de sus afluentes de la izquierda, ante todo del río Taquari, hasta Porto Manga. El desparramadero del Taquari ha llegado hasta las explanadas con relieves residuales del Chaco y recubre dichas explanadas entre las sierras-islas, con una inclinación muy débil, pero orientada en dirección contraria a la de las explanadas (fig. 4). En esta parte distal del abanico, el material se torna fino. Digitaciones de desparramaderos hon penetrado en dirección del W entre las serranias residuales. Pero, sólo una pequeña porción del material ha podido dar la vuelta atrós de estos relieves. Inmediatamente, al W de ellos, se localizon áreas de acumulación deficiente. Con el cambio de clima hacia una mayor humedad, estas depresiones se ahogaron. Hoy en día, las ocupan lagunas y lagos en las cuales entra el agua del río Paraguay durante las crecidas. Cuando bajan las aguas, una parte de ellas vuelve al río Paraguay. La comunicación se establece por medio de ciénogas en las cuales, en algunas partes, parecen canales definidos. Ciertos lagos se comunican también entre si por canales, como el Canal Pedro II que une, sobre una distancia de unos $100 \mathrm{~km}$, la Laguna Uberaba con el Lago Gaíbo. La Laguna Uberaba recibe aguas del río Paraguay por intermedio del Bracinho. Otro brazo del Paraguay llega directamente al Lago Guaiba. Además, sobre una distancia de $40 \mathrm{~km}$ aguas abajo de Porto Conceişao, el margen derecho del río Paraguay está ocupado por ciánagas desde los cuales el agua se dirige en dirección de los lagos. Otro canal más une el rio Paraguay al Lago Gaíba aguas arriba de Tres Bocas: el Riacho de Gaiba cuyo ancho es igual al del propio rio Pa. raguay. Este canal corre a veces en dirección de los lagos y, en otros casos, desde los lagos hasta el Paraguay y de un lago a otro, en función de los cambios de niveles del propio río Paraguay. En efecto muy escasa agua de los lagos lle. ga del Chaco. Esta circulación, muy lenta por la casi ausencia de pendiente, permite una amortiguación eficiente de las crecidas, pero, también provoca enormes pérdidas de agua como consecuencia de la evaporación y del alto consumo 
de la vegetación cenagosa. El déficit de escurrimiento aumenta por consecuencia. Una parte de las aguas del Lago Gaíba, durante las fuertes crecidas sigue su camino hasta el otro lago, la Laguna de Gaiba Mirim, en territorio boliviano, pero, desde allí, nunca regresan al río Paraguay: desaparecen por evaporación.

Estos lagos se caracterizan por un nivel fluctuante, de acuerdo con esta circulación muy compleja. Su extensión cambia mucho:

$\begin{array}{lll}\text { Periodo de aguas bajas } & 55 \mathrm{~km} 2 & 20 \mathrm{~km} 2 \\ \text { Periodo de aguas altas } & 105 \mathrm{~km} 2 & 40 \mathrm{~km} 2\end{array}$

En período de aguas bajas la vegetación cubre una alta proporción de la superficie fluctuante de estos lagos. Asi ocurre con la mitad en el Lago Gaíba Mirim. El Lago Gaíba es muy raso: en período de estiaje, sólo $2 \mathrm{~km}$ de su extremidad $N$ ofrecen una profundidad de 0,1-0,6 m. MINTER (1966-1972) evalúa en $1480 \mathrm{~km} 2$ la superficie de las ciénagas a lo largo del río Paraguay entre Laguna Uberaba y Tres Bocas; de las cuales $400 \mathrm{~km} 2$ los ocupan espejos de agua permanentes.

Este ejemplo presentado de manera pormenorizada, es perfectamente ilustrativo. Varios otros lagos se localizan sobre el margen derecho del río Paragudy y comunican con él, con escurrimiento alternativo: los principales son los lagos Mandioré (con distensiones extremas de 80 y $200 \mathrm{~km} 2$ en periodo de crecida mediana), do Castelo (70 km2 en aguas medianas), Cáceres (90 km2 en aguas medianas, de los cuales 30 son cubiertos de vegetación). Las ciénagas son extensas. Defluentes salen del río Paraguay en dirección de los lagos (Riacho de Conceiçao, que alimenta $220 \mathrm{~km} 2$ de ciénagas y lagunetas, Canal Tamengo que alimenta el Lago Cáceres, el Tuiviu cerca de Corumbá). El Paraguay Mirim es un "paraná", es decir, un defluente que se une nuevamente, aguas abajo, al río del cual viene. Su longitud es de sólo 122 km contra 174 por el río Paraguay.

El tipo de cauce del río Paraguay sigue alimentando ciénagas y defluentes hasta Porto Esperanga, donde se edificó el puente del ferrocarril a Bolivia, por Corumbá. Allí se termina el Pantanal típico. A pesar de escapar una parte del agua a la evaporación, por encontrarse infiltrada en los desparramaderos arenosos, podemos entender por qué el río Paraguay es tan poco caudaloso. Aguas abajo, él recorre áreas más secas, lo que no mejora su módulo y explica la escasa profundidad de su lecho al cruzar barras rocosas y los problemas de navegoción derivados de ello.

Bajo el clima relativamente húmedo de nuestra época, una cantidad impresionante del agua de la cuenca alta del río Paraguay se pierde por evapotranspiración en el Pantanal. Podemos entender fácilmente que, durante épocas más secas del Cuaternario, los caudales no fueron suficientes para permitir el exorreismo. La disposición de los abanicos, principalmente del mayor de ellos, el del río Taquari, muestra una tendencia de acumulación aluvial al ahogar el propio Pantanal. Aguas abajo de Porto Esperanga, el cauce del río Paraguay se torna alóctono a la región recorrida: el río no recibe afluentes en su margen derecho y sólo uno en el margen izquierdo, el río Apa, unos $300 \mathrm{~km}$ aguas abajo de Porto Esperansa, en línea recta. Opinamos que, a lo largo de este tramo, el rio Paragudy ha instalado su cauce recientemente, cuando el clima vuelve a ser húmedo, probablemente al inicio del Holoceno, si nos basamos sobre lo que pasó 
en Amazonia y en la Pampa Deprimida argentina, es decir, al $\mathrm{N}$ y al $\mathrm{S}$ de nuestra área de estudio. El endorreismo del Alto Paraguay es el resultado de la combinación de dos mecanismos diferentes:

- oscilaciones climáticas al nivel del continente sudamericano

- la subsidencia cuaternaria del propio Pantanal

\section{CONCLUSION}

Terminaremos enfocando algunas consecuencias de la evolución geomorfológica que acabamos de reconstruir:

- El control de las crecidas e inundaciones en el Pantanal es un problema altamente complejo. Es posible que la edificación de algunas grandes represas sobre los ríos cercanos al Pantanal, alivie la situación. Pero, tales obras tienen que ofrecer una gran capacidad de almacenamiento, y por consecuencia, serán de muy alto costo. Por otra parte, la cuenca del rio Taquari, que desempeña el mayor papel en este caso, se caracteriza por importantes superficies intensamente disecadas durante el último período seco. Ofrecen una altisima torrencialidad potencial. Si se reactivan, ellas van a producir nuevamente grandes cantidades de sedimentos, arena, limos, arcilla, que van a colmatar los embolses futuros. Ahora bien, modificaciones muy rápidas se están produciendo con la explotación del Planalto Mato-Grossense. Los mosaicos de radar muestran campos enormes, recién talados y quemados, algunos de millares de hectáreas cada uno. El cultivo mecanizado de la soya se extiende muy rápidamente. El peligro de cambios drásticos en el escurrimiento y en el arrastre aluvial se torna agudo. Una planificación al nivel regional, de tipo integrado, inspirada por la filosofía del Programa MAB de la UNESCO, es una necesidad urgente.

- Los cambios muy importantes en la morfodinámica que acabamas de demostrar y los cambios climáticos estrechamente asociados con ellos, dan a pensar que la vegetación muy especifica del Pantanal es una vegetacián pionera, recién instalada. Como en Amazonia, parece haber colonizado el Pantanal como consecuencia de la oscilación húmeda del Holoceno. Faltan los estudios biogeográficos, pero el problema debe ser planteado. Hemos enfocado la persistencia de varios rasgos de aridez de corácter relicto. Lógicamente, no pueden limitarse a los aspectos geomorfológicos. Lo mismo debe aparecer en las biocénosis, incluso en las formaciones vegetales. Su estudio es necesario para un mejor aprovechamiento de los recursos naturales y debe apoyarse sobre el estudio geomorfológico. En el presente trabajo, hemos enfocado la importancia de las aguas subterráneas superficiales en el Pantanal. Ellas se alimentan a partir de las inundaciones que afectan la parte alta de los desparramaderos. En una gran parte del área, los "vazantes" y las "corixas" recojen el agua de manantiales y son una fuente de abastecimiento indispensable para la cría del ganado. Si se edifican represas de control de las crecidas, estas aguas subterráneas no van a alimentarse más y el recurso va a desaparecer. Esto merece mucha atención. Para evitar este grave inconveniente, sería necesario realizar una ordenación del ambiente que incluye una recarga del acuífero aguas abajo de las represas. Solución técnicamente delicada $y$, de todas maneras, muy onerosa. 
El Pantanal es un ambiente muy complejo y de alta sensibilidad. Cualquiera intervención debe ser muy cuidadosamente planeada y basada sobre conocimientos que faltan todavía. Cambiar algo en su dinámica puede resultar en consecuencias indirectas muy graves. Es bien posible que funcione, en su caso, una ley ile noda o todo.... Si intentamos cambiar algo, debemos cambiarlo por completo. Eil riesgo de fracasar es grande.

\section{AGRADECIMIFNTOS}

Los tratamienros fotográficos de los mosoices $j:$ : adar fueron hechos por $D$. Blumenroeder en el laboratorio del Centre de lieographie Appliquée LA 95. La fig. 5 fué elaborada en el Servicio Cartográfico de este centro por la Sra. A. Bouzeghaia bajo la orientación de la Sra. Cl. Beller, jefe del servicio. La Documentación-base del presente trabojo fue recogida por el personal de la DIMOR de la Base de Goiánia de RADAMBRASIL, al cual dirigimos nuestres agradecimientos cariñosos. Es como consultor de RADAMBRASIL. que he tenido la oportunidad de interesarse por el Pantanal. Agradezco a mi amigo Trento Natali FILHO, Director de Geomorfologío do P.ADAMBRASIL por todo lo que hizo para facilitar mi investigación sobre el Pantanal (RADAMBRASIL, BAGOI \& DIMOR, 1979).

\section{REFERENCIAS BIBLIOGRAFICAS}

ALMEIDA, (F. M. de) \& M. A. LIMA. 1956. The West-Central Plateau and the Mato Grosso "Pantanal". XVIII th lintern. Geogr. Congress, Rio de Janeiro. Excursion Gujde-Book № 1.

CUNHA (J. da). 1943. Cobre do Jauru e lagoas alcalinas do Pantanal (Mato Grosso). Bol, Lab. Prod. Mineral, Río de Janeiro, №6.

KLAMMER, G. 1981. Landforms, cyclic erosion and deposition, and late Genozoic changes in climate in Southern Brasil. Z. fur Geomorph. 25 (2) : 146-165.

MENDES, J. C. 1957. Grutas calcárias na Serra da Bodoquena, Mato Grosso Bol. Paulista de Geogr. (25) : 70-77.

MINTER (Ministerio do Interior), DNOS, s.d.. 1966-1972. Estudos hidrológicos da banda do Alto Paraguay. Relatorio Técnico. 4 Vol. Brasilia. PNUD UNESCO.

MINISTERIO DAS MINAS E ENERGIA, DNPM, 1976. Convenio DNPM/CPRM: Proyecto Bodoquena, Relatorio final, Texto integrado, Vol. I.

RADAMBRASIL, BAGOI \& DIMOR. 1979. Estudios preliminares visando o conroles de enchentes na bacio do Alto Paraguay. Dactilografiado.

SANCHEZ, R. O. 1977. Estudio geomorfológico del Pantanal. Regionalización y sectorización geográfica de la depresión de la alta cuenca del Rio Paraguay. EDIBAP, Convenio Gob. Brasileiro-UNDP-OEA. Programas de des. Reg. de la OEA. SILVESTRE, D. F. Filho \& N. ROMEU. 1974. Característicos o potencialidades do Pantanal Matogrossense. IPEA, EST. para o Planejamento, 10.

VALVERDE, O. 1972. Fundamentos geográficos do planejamento rural do Município de Corumbá. R. Brasileira de Geogr. 34 (1): 49-144. 